\title{
MEIXNER POLYNOMIALS OF THE SECOND KIND AND QUANTUM ALGEBRAS REPRESENTING $s u(1,1)$
}

\author{
GÁBOR HETYEI
}

\begin{abstract}
We show how Viennot's combinatorial theory of orthogonal polynomials may be used to generalize some recent results of Sukumar and Hodges on the matrix entries in powers of certain operators in a representation of $s u(1,1)$. Our results link these calculations to finding the moments and inverse polynomial coefficients of certain Laguerre polynomials and Meixner polynomials of the second kind. As an immediate consequence of results by Koelink, Groenevelt and Van Der Jeugt, for the related operators, substitutions into essentially the same Laguerre polynomials and Meixner polynomials of the second kind may be used to express their eigenvectors. Our combinatorial approach explains and generalizes this "coincidence".
\end{abstract}

\section{INTRODUCTION}

In two recent papers (Hodges \& Sukumar 2007; Sukumar \& Hodges 2007), Sukumar and Hodges introduced a one-parameter family of operator algebras exhibiting a paritydependent structure, to study a quantum harmonic oscillator. The operators $R$ and $L$, arising as the half of the square of the creation and annihilation operators, respectively, generate a subalgebra representing the Lie algebra $s u(1,1)$. This representation is the direct sum of two discrete series representations. Sukumar and Hodges observed that calculating the matrix entries in the powers of $L+R$ lead to some interesting combinatorial questions. In this paper we show that these results may be generalized in such a way that the answer may be expressed in terms of moments and inverse polynomial coefficients associated to Laguerre polynomials and Meixner polynomials of the second kind.

Almost at the same time, Klimyk (2006) used discrete series representations of $s u(1,1)$ to study another model of a quantum harmonic oscillator. In Klimyk's setting, the operator $L+R$ corresponds to the momentum operator, and Klimyk (2006) noted that the eigenfunctions of this operator may be expressed via substitutions into Meixner-Pollaczek polynomials with the appropriate parameters. This observation is a consequence of the more general results of Van Der Jeugt (1997), Koelink \& Van Der Jeugt (1998) and Groenevelt \& Koelink (2002), describing the spectra of certain self-adjoint elements in an arbitrary discrete series representation of $s u(1,1)$, where

1991 Mathematics Subject Classification. Primary 81S05; Secondary 05E35, 33C45.

Key words and phrases. Quantum algebra; su(1,1); Laguerre polynomials; Meixner polynomials of the second kind. 
besides the Meixner-Pollaczek polynomials, the Laguerre polynomials and the Meixner polynomials of the first kind also appear, depending on the choice of the parameters.

In this paper we generalize and explain this remarkable "coincidence", using Viennot's (1983) combinatorial theory of orthogonal polynomials. For that purpose we will consider tridiagonal operators $T$ defined on a subspace of $\ell^{2}\left(\mathbb{Z}_{\geq 0}\right)$ that contains at least the subspace generated by the basis vectors $\left\{e_{0}, e_{1}, e_{2}, \ldots\right\}$.

In section 2 we express the matrix entries $\left\langle e_{i+d}, T^{m} e_{i}\right\rangle$ in terms of weighted Motzkin paths. Using Viennot's (1983) theory, in the case when the matrix of $T$ has nonzero off-diagonal entries wherever this is allowed, we are able to associate an orthogonal polynomial sequence $\left\{p_{n}(x)\right\}_{n \geq 0}$ to $T$ in such a way that the matrix entries $\left\langle e_{0}, T^{m} e_{0}\right\rangle$ and $\left\langle e_{d}, T^{m} e_{0}\right\rangle$ may be expressed in terms of the moments and inverse polynomial coefficients associated to $\left\{p_{n}(x)\right\}_{n \geq 0}$. Applications of these general results to the discrete series representations of $s u(1,1)$ follow in section 3 .

In section 4 we see that for those tridiagonal operators $T$ that have nonzero offdiagonal matrix entries wherever this is allowed, the same orthogonal polynomial sequence $\left\{p_{n}(x)\right\}_{n \geq 0}$ as in section 2 may be associated to $T$ to express its (potential) eigenvectors. For the discrete series representations of $s u(1,1)$ we thus recover the necessity part of the above mentioned results of Van Der Jeugt (1997), Koelink \& Van Der Jeugt (1998) and Groenevelt \& Koelink (2002).

The ideas presented in this paper also provide a combinatorial framework to discuss some representations of other Lie (super)algebras over $\ell^{2}\left(\mathbb{Z}_{\geq 0}\right)$. In the concluding section 5 we outline a sample application. The other example in section 5 indicates that we do not have to limit ourself to self-adjoint operators at this level of investigation. It seems harder to replace the Hilbert space $\ell^{2}\left(\mathbb{Z}_{\geq 0}\right)$ with another Hilbert space, as in that case one would be facing the dilemma, what notion should replace the notion of the associated orthogonal polynomial sequences. Finding such a generalization seems an interesting challenge for future research.

\section{Preliminaries}

1.1. Viennot's combinatorial proof of Favard's theorem. Concerning orthogonal polynomials, in this paper we follow Viennot's (1983) notation and terminology, but sometimes we complement the facts stated therein with results cited from Chihara's (1978) classical work. Viennot's work (1983) is currently out of print but available on the author's webpage. Some of his most important results were also communicated by Stanton (2003), a recommended source for those readers whose French is not fluent.

The direct way to define an orthogonal polynomial sequence (OPS) $\left\{p_{n}(x)\right\}_{n \geq 0}$ is to provide a linear form $f: \mathbb{C}[x] \rightarrow \mathbb{C}$ and postulate the following three axioms:

(i) for all $n, p_{n}(x)$ is a polynomial of degree $n$, 
(ii) $f\left(p_{m}(x) p_{n}(x)\right)=0$ if $m \neq n$,

(iii) for all $n, f\left(p_{n}(x)^{2}\right) \neq 0$.

The map $f$ is called a moment functional. Whenever an OPS exists, each of its elements is determined up to a non-zero constant factor, see the corollary of theorem 2.2 in ch. I of Chihara's (1978) book.

An equivalent way define an OPS is by means of Favard's theorem, see theorems 4.1 and 4.4 in ch. I of Chihara's (1978) book. This states that a sequence of monic polynomials $\left\{p_{n}(x)\right\}_{n \geq 0}$ is an orthogonal polynomial sequence, if and only if it satisfies the initial conditions

$$
p_{0}(x)=1, p_{1}(x)=x-b_{0},
$$

and a two-term recurrence formula

$$
p_{n+1}(x)=\left(x-b_{n}\right) p_{n}(x)-\lambda_{n} p_{n-1}(x) \text { for } n \geq 1,
$$

where the numbers $b_{n}$ and $\lambda_{n}$ are constants and $\lambda_{n} \neq 0$ for $n \geq 1$. The above form appears in theorem 9 of ch. I in Viennot's (1983) book, in Chihara's (1978) work the indices are shifted. The original proof of Favard's theorem provides only a recursive description of the moment associated functional $f$. In proposition 17 of ch. I, Viennot (1983) gave an explicit combinatorial description by expressing the moments $\mu_{n}:=$ $f\left(x^{n}\right)$ as sums of weighted Motzkin paths. A Motzkin path of length $n$, as defined in def. 15 of ch. I by Viennot (1983), is a path $\omega=\left(s_{0}, \ldots, s_{n}\right)$ in $\mathbb{Z} \times \mathbb{Z}$ from $s_{0}$ to $s_{n}$ such that the second coordinate of all $s_{i}$ 's is non-negative, and each step $\left(s_{i}, s_{i+1}\right)$ is either a northeast step $(1,1)$ or an east step $(1,0)$ or a southeast step $(1,-1)$. Viennot (1983) introduces the following valuation: the weight $v(\omega)$ of $\omega$ is the product of the weights of its steps $\left(s_{i-1}, s_{i}\right)$, where each northeast step has weight 1 , each east step at level $k$ has weight $b_{k}$ and each southeast step starting at level $k$ has weight $\lambda_{k}$. Here the level is the second coordinate of the lattice point. He then defines $\mu_{n}:=\sum_{\omega} v(\omega)$ as the total weight of all Motzkin paths $\omega$ from $(0,0)$ to $(n, 0)$.

Theorem 1.1 (Viennot (1983)). Let $\left\{p_{n}(x)\right\}_{n \geq 0}$ be a sequence of monic polynomials, given by (1.1) and (1.2) and let $f: \mathbb{C}[x] \rightarrow \mathbb{C}$ be the linear map given by $f\left(x^{n}\right):=\mu_{n}$. Then for all $n, k, \ell \geq 0$ we have

$$
f\left(x^{n} p_{k}(x) p_{\ell}(x)\right)=\lambda_{1} \cdots \lambda_{\ell} \sum_{\omega} v(\omega)
$$

where the summation is over all Motzkin paths of length $n$ from level $k$ to level $\ell$.

Substituting $n=0$ in theorem 1.1 yields the more difficult implication of Favard's theorem. A generalization of theorem 1.1] is theorem 1 in ch. III of Viennot's (1983) work, which allows the computation of the inverse polynomials of a system of monic polynomials $\left\{p_{n}(x)\right\}_{n \geq 0}$ given by (1.1) and (1.2), even if some scalars $\lambda_{n}$ are zero (and thus $\left\{p_{n}(x)\right\}_{n \geq 0}$ is not an OPS). The inverse polynomials $q_{n}(x):=\sum_{i=0}^{n} q_{n, i} x^{i}$ are defined by $x^{n}=\sum_{i=0}^{n} q_{n, i} p_{i}(x)$.

Theorem 1.2 (Viennot (1983)). Let $\left\{p_{n}(x)\right\}_{n \geq 0}$ be a system of monic polynomials defined by (1.1) and (1.2) for some numbers $\left\{b_{n}\right\}_{n \geq 0}$ and $\left\{\lambda_{n}\right\}_{n \geq 1}$. The coefficient $q_{n, k}$ 
of $x^{k}$ in the inverse polynomial $q_{n}(x)$ is then the total weight of all weighted Motzkin paths of length $n$ starting at $(0,0)$ and ending at $(n, k)$.

1.2. Viennot's "histoires", Laguerre and Meixner polynomials. Sometimes models of even deeper combinatorial interest may be built, using Viennot's (1983) second valuation of Motzkin paths, defined as follows. Given the sequences of numbers $\left\{a_{k}\right\}_{k \geq 0}$, $\left\{b_{k}\right\}_{k \geq 0}$ and $\left\{c_{k}\right\}_{k \geq 1}$, we define the weight $v_{1}(\omega)$ of a Motzkin path $\omega$ as the product of the weights $v_{1}\left(s_{i-1}, s_{i}\right)$ of its steps, such that each northeast (resp. east, resp. southeast) step starting at level $k$ has weight $a_{k}$ (resp. $b_{k}$, resp. $c_{k}$ ). Setting

$$
\lambda_{k}=a_{k-1} c_{k} \quad \text { for } k \geq 1,
$$

for a Motzkin path $\omega$ from $(0,0)$ to $(n, 0), v_{1}(\omega)$ is equal to $v(\omega)$, as defined in subsection 1.1, because each northeast step starting at some level $k-1$ may be matched to the first subsequent southeast step starting at level $k$. More generally, for a Motzkin path $\omega$ starting at level $k$ and ending at level $l$, we have

$$
v(\omega)= \begin{cases}\sum_{\omega} v_{1}(\omega) & \text { if } k=l, \\ a_{k} a_{k+1} \cdots a_{l-1} \sum_{\omega} v_{1}(\omega) & \text { if } k<l \\ c_{k} c_{l-1} \cdots c_{l+1} \sum_{\omega} v_{1}(\omega) & \text { if } k>l\end{cases}
$$

see lemma 1 in ch. II of Viennot (1983). Algebraically, eq. (1.3) corresponds to switch from the three term recurrence relation for monic orthogonal polynomials to a general three term recurrence relation. The combinatorial interest in $v_{1}$ arises when the sequences $\left\{a_{k}\right\}_{k \geq 0},\left\{b_{k}\right\}_{k \geq 0}$ and $\left\{c_{k}\right\}_{k \geq 1}$ consist of non-negative integers, allowing us to think of these weights as making choices from a set of options. In such situations Viennot (1983) defines a story ("histoire") as a pair $\left(\omega ;\left(p_{1}, \ldots, p_{n}\right)\right)$ of a Motzkin path $\omega=\left(s_{0}, \ldots, s_{n}\right)$ and a sequence $\left(p_{1}, \ldots, p_{n}\right)$ of positive integers satisfying $1 \leq p_{i} \leq v_{1}\left(s_{i-1}, s_{i}\right)$ for $1 \leq i \leq n$. Clearly the moment $\mu_{n}$ is the number of stories of length $n$. Thus, in such situations, we may replace the weighted lattice path model with a model that involves enumerating (non-weighted) combinatorial objects. In particular, permutation enumeration models of the (monic) Laguerre polynomials and of the Meixner polynomials of the second kind (discussed below) may be obtained by specializing Viennot's (1983) bijection between his "histoires de Laguerre" of length $n$ associated to the valuation

$$
a_{k}=k+1, \quad b_{k}=2 k+2 \quad \text { for } k \geq 0 ; \quad c_{k}=k+1 \quad \text { for } k \geq 1,
$$

and the permutations of the set $\{1, \ldots, n+1\}$. For the details we refer to Viennot's (1983) work.

The (monic) Laguerre polynomials $L_{n}^{(\alpha)}(x)$ are the OPS defined by (1.1) and (1.2) where $b_{n}=2 n+\alpha+1$ and $\lambda_{n}=n(n+\alpha)$, see $\S 5$ in ch. II of Viennot's (1983) work. The associated moments are given in (31') of ch. II by Viennot's (1983):

$$
\mu_{n}=(\alpha+1)_{n}=(\alpha+1)(\alpha+2) \cdots(\alpha+n) .
$$

The combinatorial model mentioned above is associated to the case $\alpha=1$ yielding large Laguerre stories. For general $\alpha$ we have weighted Laguerre stories, for $\alpha=0$ we obtain restricted Laguerre stories by limiting $b_{k}^{\prime}$ to $k$ above. The restricted Laguerre 
stories are thus a subset of the large Laguerre stories and correspond bijectively to the permutations $\sigma \in S_{n+1}$ with $\sigma(1)=n+1$.

Another class of particular interest to us are the Meixner polynomials of the first kind $m_{n}(x ; \delta, \eta):=(c-1)^{n} / c^{n} \hat{m}_{n}(x ; \beta, c)$, whose monic variant $\left\{\hat{m}_{n}(x ; \beta, c)\right\}_{n \geq 0}$ is defined by (1.1) and (1.2) where

$$
b_{n}=\frac{(1+c) n+\beta c}{1-c} \text { and } \quad \lambda_{n}=\frac{c n(n+\beta-1)}{(1-c)^{2}} .
$$

Viennot (1983) (see (54") in ch. 2) )has a combinatorial proof of the fact that the moments of the corresponding linear functional are given by

$$
\mu_{n}(\beta, c)=(1-c)^{\beta} \sum_{k \geq 0} k^{n} c^{k} \frac{(\beta)_{k}}{k !} .
$$

Here $(\beta)_{k}=\beta(\beta+1) \cdots(\beta+k-1)$.

Finally we will be interested in the (monic) Meixner polynomials of the second kind $M_{n}(x ; \delta, \eta)$, defined by (1.1) and (1.2) where

$$
b_{n}=(2 n+\eta) \delta \quad \text { and } \quad \lambda_{n}=\left(\delta^{2}+1\right) n(n+\eta-1) .
$$

A combinatorially interesting special case is $\delta=0$, implying $b_{n}=0$ and $\lambda_{n}=n(n+\eta-1)$. The moments associated to $\left\{M_{n}(x ; \delta, \eta)\right\}_{n \geq 0}$ may be expressed using the Motzkin paths associated to the Laguerre polynomials $\left\{L_{n}^{(\eta-1)}(x)\right\}_{n \geq 0}$ subject to the restriction that no east step occurs. Motzkin paths with no east steps are also known as Dyck paths. Using the permutation-enumeration model mentioned above, Viennot (1983) (in ch. 2, example 21) shows that $\mu_{n}(0, \eta)$ is given by

$$
\mu_{n}(0, \eta)=\sum_{\sigma \in Z_{n}} \eta^{\mathrm{s}(\sigma)}
$$

Here $Z_{n}$ is the set of alternating or zig-zag permutations of $\{1,2, \ldots, n$,$\} , starting and$ ending with a rise, and $\mathrm{s}(\sigma)$ is the number of left-to-right minima ("éléments saillants") $\sigma(i)$, defined by $\sigma(i)=\min \{\sigma(1), \sigma(2), \ldots, \sigma(i)\}$. A generating function for the numbers $\mu_{2 n}(0, \eta)$ for positive integer $\eta$ was already computed by Carlitz (1959). He considered the polynomials $f_{n}^{(k)}(x)=(-\mathbf{i})^{n} M_{n}(\mathbf{i} x ; 0, k)$ for $\mathbf{i}=\sqrt{-1}$, but using the substitution $x \mapsto x / \mathbf{i}$ we may recover the moments $\mu_{2 n}(0, \eta)$. In the next lemma we state Carlitz' (1959) result (see his formulas (9.3) and (9.13)) modified for our purposes, together with the outline of a combinatorial proof that extends its validity to all numbers $\eta$.

Lemma 1.3. For all $\eta \neq 0$ the moments of $\left\{M_{n}(x ; 0, \eta)\right\}_{n \geq 0}$ satisfy

$$
\mu_{2 n}(0, \eta)=E_{2 n}^{(\eta)} \quad \text { where } \quad \sec ^{\eta}(t)=\sum_{n \geq 0} E_{2 n}^{(\eta)} \frac{t^{2 n}}{(2 n) !} .
$$

Proof. Let $E_{2 n, k}$ denote the number of those zig-zag permutations on $2 n$ elements that start and end with a rise and have $k$ left-to-right minima. Since 1 is the rightmost 
left-to-right minimum, using the decomposition $\sigma(1) \cdots \sigma(2 n)=\sigma(1) \cdots 1 \cdots \sigma(2 n)$ we have the recursion formula

$$
E_{2 n, k}=\sum_{m=0}^{n-1}\left(\begin{array}{c}
2 n-1 \\
2 m
\end{array}\right) E_{2 m, k-1} \cdot T_{2 n-2 m-1} .
$$

Here $T_{2 n-2 m-1}$ counts the number of zig-zag permutations on $2 n-2 m-1$ elements, starting with a descent and ending with a rise. Introducing $\phi_{k}(t):=\sum_{n \geq 0} E_{2 n, k} t^{2 n} /(2 n)$ ! we obtain the recursion

$$
\phi_{k}(t)=\int_{0}^{t} \phi_{k-1}(u) \tan (u) d u \text {. }
$$

Using this, we may show by induction that $\phi_{k}(t)=(-\ln \cos (t))^{k} / k$ !. Thus, by (1.10), $\mu_{2 n}(0, \eta)$ is the coefficient of $t^{2 n} /(2 n)$ ! in

$$
\sum_{k \geq 0}(-1)^{k} \ln \cos (t)^{k} \cdot \eta^{k} / k !=\exp (-\eta \ln \cos (t))=\sec (t)^{\eta} .
$$

A variant of the Meixner polynomials of the second kind are the Meixner-Pollaczek polynomials $\left\{P_{n}^{(\lambda)}(x ; \phi)\right\}_{n \geq 0}$, defined by (1.7.1) in the Askey-Wilson scheme by Koekoek \& Swarttouw (1998). We should note for future reference that the two OPS are essentially the same. The formula connecting them is (3.22) in ch. VI of Chihara's (1978) work:

$$
P_{n}^{\lambda}(x ; \phi)=\frac{\sin ^{n} \phi}{n !} M_{n}(2 x ; \delta, 2 \lambda), \quad \delta=\cot \phi, \quad 0<\phi<\pi .
$$

Chihara (1978) does not use the adjective "Meixner-Pollaczek", but gives the same recurrence for them in (5.13) of his ch. VI as the recurrence (1.7.3) of Koekoek \& Swarttouw (1998). Setting $\phi=\pi / 2$ in (1.12) leads to $\delta=0$. According to (1.7.4) of Koekoek \& Swarttouw (1998), the normalized version of $P_{n}^{\lambda}(x ; \phi)$ is $n ! /(2 \sin \phi)^{n} P_{n}^{\lambda}(x, \phi)$. As a consequence of eq. (1.12) we obtain the following.

Corollary 1.4. For $0<\phi<1$, the normalized version of the Meixner-Pollaczek polynomials $\left\{P_{n}^{\lambda}(x ; \phi)\right\}_{n \geq 0}$ is $\left\{2^{-n} M_{n}(2 x, \cot \phi, 2 \lambda)\right\}_{n \geq 0}$.

Finally we review the inverse polynomials of $\left\{L_{n}^{(\alpha)}(x)\right\}_{n \geq 0},\left\{\hat{m}_{n}(x ; \beta, c)\right\}_{n \geq 0}$ and $\left\{M_{n}(x ; \delta, \eta)\right\}_{n \geq 0}$. All three OPS are examples of Sheffer orthogonal polynomials defined as polynomials $\left\{p_{n}(x)\right\}_{n \geq 0}$, given by a generating function

$$
\sum_{n \geq 0} p_{n}(x) \frac{t^{n}}{n !}=f(t) \exp (x g(t)) .
$$

As noted in (11) and (12) of ch. III in Viennot's (1983) work, the inverse polynomials $\left\{q_{n}(x)\right\}_{n \geq 0}$ of a Sheffer OPS $\left\{p_{n}(x)\right\}_{n \geq 0}$ form a Sheffer OPS, given by

$$
\sum_{n \geq 0} q_{n}(x) \frac{t^{n}}{n !}=\frac{1}{f\left(g^{\langle-1\rangle}(t)\right)} \exp \left(x g^{\langle-1\rangle}(t)\right) \text {. }
$$

Here $g^{\langle-1\rangle}(t)$ stands for the compositional inverse of $g(t)$. 
1.3. Discrete series representations of $s u(1,1)$ and two applications. In this subsection we review the discrete series representations of the Lie algebra $s u(1,1)$, together with two applications of them in models of a quantum oscillator. Our notation will be close to the one used by Groenevelt \& Koelink (2002).

The Lie algebra $s u(1,1)$ has the generators $H, B, C$, subject to the commutation relations $[H, B]=2 B,[H, C]=-2 C$ and $[B, C]=H$. According to section 6.4 in the work of Vilenkin and Klimyk (1991), there are four classes of irreducible unitary representations of $s u(1,1)$ : the positive and negative discrete series representations, the principal series representations and the complementary series representations. In this paper we will focus on the two discrete series representations, defined on the representation space $\ell^{2}\left(\mathbb{Z}_{\geq 0}\right)$, whose orthonormal basis vectors we will denote by $\left\{e_{n}\right\}_{n \geq 0}$. The positive discrete series representations $\pi_{k}^{+}$are labeled by $k>0$. The action is given by

$$
\begin{aligned}
\pi_{k}^{+}(H) e_{n} & =2(k+n) e_{n}, \\
\pi_{k}^{+}(B) e_{n} & =\sqrt{(n+1)(2 k+n)} e_{n+1} \text { and } \\
\pi_{k}^{+}(C) e_{n} & =-\sqrt{n(2 k+n-1)} e_{n-1},
\end{aligned}
$$

see eq. (2.2) of Groenevelt \& Koelink (2002). The negative discrete series representations $\pi_{k}^{-}$are labeled by $k>0$. The action is given by

$$
\begin{aligned}
\pi_{k}^{-}(H) e_{n} & =-2(k+n) e_{n}, \\
\pi_{k}^{-}(B) e_{n} & =-\sqrt{n(2 k+n-1)} e_{n-1} \text { and } \\
\pi_{k}^{-}(C) e_{n} & =\sqrt{(n+1)(2 k+n)} e_{n+1},
\end{aligned}
$$

see eq. (2.3) of Groenevelt \& Koelink (2002). The principal series and complementary series representations are defined on $\ell^{2}(\mathbb{Z})$, a different Hilbert space. Koelink \& Van Der Jeugt (1998) (proposition 3.1, rephrased) have expressed the eigenvectors of $\pi_{k}^{+}(c$. $H+B-C)$ for an arbitrary $c \in \mathbb{R}$ satisfying $|c|<1$ in terms of the orthonormal Meixner-Pollaczek polynomials $p_{n}^{(\lambda)}(x ; \phi)$, given by

$$
p_{n}^{(\lambda)}(x ; \phi)=\sqrt{\frac{n !}{\Gamma(n+2 \lambda)}} P_{n}^{(\lambda)}(x ; \phi)
$$

where the polynomials $P_{n}^{(\lambda)}(x ; \phi)$ are the Meixner-Pollaczek polynomials.

Theorem 1.5 (Koelink \& Van Der Jeugt). Introducing $X_{\phi}=-\cos \phi H+B-C$, the vector

$$
v(x):=\sum_{n \geq 0} p_{n}^{(k)}(x ; \phi) e_{n}
$$

is a generalized eigenvector for $\pi_{k}^{+}\left(X_{\phi}\right)$, with eigenvalue $2 x \sin \phi$.

An analogous result for $c=1$ was worked out by Van Der Jeugt (1997), leading to Laguerre polynomials, who also mentions (in his section VIII) that the case $|c|>1$ leads to Meixner polynomials of the first kind. The extension of Theorem 1.5 to negative discrete series representations is stated in proposition 3.1 of Groenevelt \& Koelink (2002). An excellent source treating all cases simultaneously is ch. 3 of Groenevelt's thesis (2004). 
Klimyk (2006) used the positive series representations of $s u(1,1)$ to study a model of a quantum oscillator. He defines $s u(1,1)$ as the Lie algebra generated by $J_{0}, J_{+}, J_{-}$, subject to the commutation relations $\left[J_{0}, J_{+}\right]=J_{+},\left[J_{0}, J_{-}\right]=-J_{-}$and $\left[J_{-}, J_{+}\right]=2 J_{0}$. This definition is equivalent to the above one, via setting $H:=2 J_{0}, B:=J_{+}$and $C:=-J_{-}$. Klimyk (2006) realizes the orthonormal basis vectors $\left\{e_{n}\right\}_{n \geq 0}$ as polynomials $e_{n}^{k}(y):=\left(\frac{(2 k+n-1) !}{n !}\right)^{1 / 2} y^{n}$ in the single variable $y$, and the Hilbert space $\ell^{2}\left(\mathbb{Z}_{\geq 0}\right)$ is realized as the closure of the space of all polynomials in a single variable $y$, whereas the generators $J_{0}, J_{+}, J_{-}$of $s u(1,1)$ become differential operators. One of Klimyk's (2006) observations (eq. (17)) is that the eigenfunctions of the momentum operator $J_{1}:=\frac{1}{2}\left(J_{+}+J_{-}\right)$are of the form

$$
\psi_{p}(y)=\sum_{n \geq 0} P_{n}^{(k)}(p ; \pi / 2) y^{n} .
$$

with the Meixner-Pollaczek polynomials $P_{n}^{(k)}(p ; \pi / 2)$. Obviously, (1.17) may be obtained from Theorem 1.5 by substituting $\phi=\pi / 2$. By eq. (1.7.11) of Koekoek \& Swarttouw (1998), eq. (1.17) is equivalent to $\psi_{p}(y)=(1-\mathbf{i} y)^{-k+\mathbf{i} \cdot p}(1+\mathbf{i} y)^{-k-\mathbf{i} \cdot p}$.

Sukumar \& Hodges (2007) considered another model of a quantum oscillator, using (besides others) the operators $L, R, S$, acting on $\ell^{2}\left(\mathbb{Z}_{\geq 0}\right)$ as follows:

$$
\begin{aligned}
L e_{2 n} & =\sqrt{(2 n+1+\alpha)(2 n+2))} / 2 \cdot e_{2 n+2} \\
L e_{2 n-1} & =\sqrt{(2 n+1+\alpha)(2 n))} / 2 \cdot e_{2 n+1} \\
R e_{2 n+2} & =\sqrt{(2 n+1+\alpha)(2 n+2))} / 2 \cdot e_{2 n} \\
R e_{2 n+1} & =\sqrt{(2 n+1+\alpha)(2 n))} / 2 \cdot e_{2 n-1} \\
S e_{n} & =(2 n+1+\alpha) / 2 \cdot e_{n}
\end{aligned}
$$

The parameter $\alpha \in \mathbb{R}$ is assumed to satisfy $|\alpha| \leq 1$. As noted by Sukumar \& Hodges (2007) in eq. (2.1), the operators $L, R, S$ obey the same commutation rules as the generators $B,-C, H$ above, thus we obtain a representation $\pi$ of $s u(1,1)$ by setting

$$
\pi(B):=L, \quad \pi(C):=-R, \quad \pi(H):=S .
$$

This representation may be written as a direct sum of two representations by considering the restrictions of the operators $L, R, S$ to the closure of the subspaces generated by $\left\{e_{2 n}\right\}_{n \geq 0}$ and $\left\{e_{2 n+1}\right\}_{n \geq 0}$, respectively. Introducing $\pi_{\beta}$ to denote the representation of $s u(1,1)$ given by

$$
\begin{aligned}
\pi_{\beta}(B) e_{n} & =\sqrt{(n+1)(n+\beta)} e_{n+1}, \\
\pi_{\beta}(C) e_{n} & =-\sqrt{n(n-1+\beta)} e_{n-1}, \\
\pi_{\beta}(H) e_{n} & =2(n+\beta / 2) e_{n},
\end{aligned}
$$

it is not difficult to see that first restriction is equivalent to $\pi_{(\alpha+1) / 2}$, whereas the second restriction is equivalent to $\pi_{(\alpha+3) / 2}$. Comparing (1.20) with (1.15) we obtain $\pi_{\beta}=\pi_{\beta / 2}^{+}$ for $\beta>0$.

Corollary 1.6. For $\alpha \in(-1,1]$, the representation $\pi$ of $s u(1,1)$ given by $(1.19)$ is isomorphic to the direct sum of the positive discrete discrete series representations $\pi_{(\alpha+1) / 4}^{+}$ and $\pi_{(\alpha+3) / 4}^{+}$. 
Remark 1.7. For $\beta=0$, we get $\pi_{0}(B) e_{0}=\pi_{0}(C) e_{0}=\pi_{0}(H) e_{0}=0$, thus we may decompose $\pi_{0}$ as a direct sum of zero acting on the linear span of $e_{0}$ and of the restriction of $\pi_{0}$ acting on the orthogonal complement $e_{0}^{\perp}$ of $e_{0}$. By shifting basis vector indices in $e_{0}^{\perp}$ we may show that the restriction of $\pi_{0}$ to $e_{0}^{\perp}$ is equivalent to $\pi_{2}=\pi_{1}^{+}$.

It should be emphasized that corollary [1.6 is applicable only to the representation of $s u(1,1)$, induced by the operators $L, R, S$. In the work of Sukumar \& Hodges (2007) these operators are expressed in terms of the annihilation operator $A$ and its adjoint $A^{\dagger}$. The algebra generated by $A$ and $A^{\dagger}$ is a representation of a Lie superalgebra properly containing $s u(1,1)$. In this paper we focus on the combinatorial statements related to the operators $L, R, S$ and generalizations of these. In particular, Hodges \& Sukumar (2007) show (see eq. (5.2)) that for $\alpha=1$ we have

$$
\left\langle e_{0},(L+R)^{2 m} e_{0}\right\rangle=E_{2 m} \quad \text { and } \quad\left\langle e_{1},(L+R)^{2 m} e_{1}\right\rangle=T_{2 m+1} \quad \text { for all } m \geq 0 .
$$

Here the numbers $\left\{E_{2 m}\right\}_{m \geq 0}$ resp. $\left\{T_{2 m+1}\right\}_{m \geq 0}$ are the secant resp. tangent numbers, given by

$$
\sec (x)=\sum_{m \geq 0} \frac{E_{2 m}}{(2 m) !} x^{2 m} \text { and } \tan (x)=\sum_{m \geq 0} \frac{T_{2 m+1}}{(2 m+1) !} x^{2 m+1} .
$$

At the light of corollary 1.6, eq. (1.21) is equivalent to

$$
\left\langle e_{0}, \pi_{1 / 2}^{+}(B-C)^{m} e_{0}\right\rangle=E_{2 m} \quad \text { and } \quad\left\langle e_{0}, \pi_{1}^{+}(B-C)^{m} e_{0}\right\rangle=T_{2 m+1} .
$$

\section{MAtrix Entries of tridiagonal operators on $\ell^{2}\left(\mathbb{Z}_{\geq 0}\right)$}

Definition 2.1. Consider the complex Hilbert space $\ell^{2}\left(\mathbb{Z}_{\geq 0}\right)$ with orthonormal basis vectors $e_{0}, e_{1}, e_{2}, \ldots$ We call a linear operator $T: D(T) \subset \ell^{2}\left(\mathbb{Z}_{\geq 0}\right) \rightarrow \ell^{2}\left(\mathbb{Z}_{\geq 0}\right)$, defined on at least the linear span of $\left\{e_{0}, e_{1}, e_{2}, \ldots,\right\}$, a tridiagonal operator, if there exists real numbers $\left\{l_{n}\right\}_{n \geq 0},\left\{d_{n}\right\}_{n \geq 0}$ and $\left\{u_{n}\right\}_{n \geq 0}$ such that $T e_{0}=d_{0} e_{0}+l_{0} e_{1}$ and, for each $n \geq 1$, we have $T e_{n}=u_{n} e_{n-1}+d_{n} e_{n}+l_{n} e_{n+1}$.

Equivalently, the restriction of $T$ to the linear span of $\left\{e_{0}, e_{1}, e_{2}, \ldots,\right\}$ may be represented by a tridiagonal matrix

$$
\left(\begin{array}{ccccccc}
d_{0} & u_{0} & 0 & 0 & 0 & 0 & \ldots \\
l_{0} & d_{1} & u_{1} & 0 & 0 & 0 & \ldots \\
0 & l_{1} & d_{2} & u_{2} & 0 & 0 & \ldots \\
0 & 0 & l_{2} & d_{3} & u_{3} & 0 & \ldots \\
\vdots & & \ddots & \ddots & \ddots & \ddots &
\end{array}\right)
$$

such that all entries are real numbers.

The letters $\left\{l_{n}\right\}_{n \geq 0},\left\{u_{n}\right\}_{n \geq 0}$ and $\left\{d_{n}\right\}_{n \geq 0}$ should remind the reader of the words "lower", "upper", and "diagonal", as it is customary in numerical analysis. We define 
the $L D U$-decomposition of the tridiagonal operator $T$ as the sum of operators $T=$ $L+D+U$, where the operators $L, D, U$ are given by $U e_{0}=0$ and

$$
L e_{n}=l_{n} e_{n+1}, \quad U e_{n+1}=u_{n} e_{n} \quad D e_{n}=d_{n} e_{n} \quad \text { for } n \geq 0 .
$$

Remark 2.2. A it is a fortunate coincidence that the operator $L$ used by Sukumar \& Hodges (2007) corresponds to the operator $L$ in this setting, modulo corollary 1.6. The choice of $D$ will depend on what we want to compute: to recover the formulas regarding the matrix entries of $L+R$ and $L+R+S$ in the work of Sukumar \& Hodges (2007) and Hodges \& Sukumar (2007) we would need to equate $D$ to zero or to the appropriate restriction of $S$.

The following lemma may be considered as a generalization of equations (4.2) and (4.3) in Hodges \& Sukumar (2007). A reader making this comparison should note that, for technical reasons, we read the words $X$ consisting of the letters $L, D, U$ right-to-left.

Lemma 2.3. Let $X$ be a word of length $m$ consisting of the letters $L, D, U$, where $T=L+D+U$ is the $L D U$-decomposition of a tridiagonal operator $T: D(T) \subset$ $\ell^{2}\left(\mathbb{Z}_{\geq 0}\right) \rightarrow \ell^{2}\left(\mathbb{Z}_{\geq 0}\right)$ and let $i \geq 0$ and $d \geq-i$ be integers. Associate to the pair $(X, i)$ a lattice path starting at $(0, i)$ such that each $L$ is a southeast step, each $U$ is a northeast step, each $D$ is an east step, and we read the letters in $X$ right-to-left. Then $\left\langle e_{i+d}, X e_{i}\right\rangle$ is not zero only if the lattice path associated to $(X, i)$ is a Motzkin path of length $m$ from $(0, i)$ to $(m, i+d)$.

Proof. Applying $X$ to $e_{i}$ involves applying the operators $L, D, U$ in it, reading $X$ from right to left. While applying these operators, at every instance of the calculation our partial result is a scalar multiple of a single basis vector. Each $L$ increases the index of this basis vector by 1 , each $U$ decreases it by 1 and each $D$ leaves the index unchanged. Thus the end result is either zero or a scalar multiple of $e_{i+d}$ where $d$ is the difference between the number of $L_{\mathrm{s}}$ and $U_{\mathrm{s}}$ in $X$ (and may be negative). If at any instance the number of $U_{\mathrm{s}}$ read exceeds the number of $L_{\mathrm{s}}$ by more than $i$ then we get zero by $U e_{0}=0$. Therefore, if $\left\langle e_{i+d}, X e_{i}\right\rangle \neq 0$ then $(X, i)$ must represent a Motzkin path starting at $(0, i)$ and necessarily ending at $(m, i+d)$.

The next lemma generalizes equations (4.6) and (4.7) of Hodges \& Sukumar (2007). Our formulas look somewhat different mainly because of our convention of reading $X$ right-to-left.

Lemma 2.4. For all $p, i \geq 0$ and $d \geq-i$ we have

$$
\begin{aligned}
\left\langle e_{i+d}, X D L^{p} e_{i}\right\rangle & =d_{p+i} \cdot\left\langle e_{i+d}, X L^{p} e_{i}\right\rangle \quad \text { and } \\
\left\langle e_{i+d}, X U L^{p+1} e_{i}\right\rangle & =l_{p+i} u_{p+i} \cdot\left\langle e_{i+d}, X L^{p} e_{i}\right\rangle .
\end{aligned}
$$

The proof is immediate. Using eq. (1.4) and lemmas 2.3 and 2.4 we may express each $\left\langle e_{i+d}, T^{m} e_{i}\right\rangle$ in terms of a total weight of weighted Motzkin paths. 
Theorem 2.5. Let $i \geq 0$ and $d \geq-i$ be integers, $T: D(T) \subset \ell^{2}\left(\mathbb{Z}_{\geq 0}\right) \rightarrow \ell^{2}\left(\mathbb{Z}_{\geq 0}\right) a$ tridiagonal operator and $T=L+D+U$ its $L D U$-decomposition. Then we have

$$
\left\langle e_{i+d}, T^{m} e_{i}\right\rangle= \begin{cases}\sum_{\omega} v(\omega) & \text { if } d=0, \\ l_{i} l_{i+1} \cdots l_{i+d-1} \sum_{\omega} v(\omega) & \text { if } d>0, \\ u_{i-1} u_{i-2} \cdots u_{d} \sum_{\omega} v(\omega) & \text { if } d<0 .\end{cases}
$$

Here $\sum_{\omega} v(\omega)$ is the total weight of all weighted Motzkin paths of length $m$ starting at $(0, i)$, ending at $(m, i+d)$ such that each northeast step has weight 1 , each southeast step starting at level $n$ has weight $\lambda_{n}=l_{n-1} u_{n-1}$, and each east step at level $n$ has weight $d_{n}$. In particular, if $D$ is identically zero then $\sum_{\omega} v(\omega)$ is the total weight of all weighted Dyck paths of length $m$ starting at $(0, i)$, ending at $(m, i+d)$ such that each northeast step has weight 1 and each southeast step starting at level $n$ has weight $\lambda_{n}=l_{n-1} u_{n-1}$.

As a consequence of theorem 1.1, setting $d=0$ and $i=0$ in theorem 2.5 yields the following algebraic statement.

Corollary 2.6. If $l_{n} u_{n} \neq 0$ for all $n \geq 0$ then for all $m,\left\langle e_{0}, T^{m} e_{0}\right\rangle$ is the mth moment of the functional associated to the OPS given by (1.1) and (1.2) where $b_{n}=d_{n}$ and $\lambda_{n}=l_{n-1} u_{n-1}$.

Remark 2.7. As noted in the Preliminaries, an OPS and the associated moments determine each other only up to a constant factor. In corollary 2.6 we understand that the moments were defined as total weights of weighted Motzkin paths as in theorem 1.1. If we obtain the moments by some other means, we always need to check whether an adjustment by a constant factor is necessary. It suffices to check whether $\mu_{0}=1$ is satisfied.

When we apply theorem 2.5 to $d \neq 0$, theorem 1.2 becomes useful, at least for the case $i=0$.

Theorem 2.8. For all $m, d \geq 0$ we have

$$
\left\langle e_{d}, T^{m} e_{0}\right\rangle=l_{0} l_{1} \cdots l_{d-1} \mu_{m, d},
$$

where $\mu_{m, d}$ is the coefficient of $x^{d}$ in the degree $m$ inverse polynomial for system of monic polynomials given by (1.1) and (1.2) where $b_{n}=d_{n}$ and $\lambda_{n}=l_{n-1} u_{n-1}$.

\section{MATRIX ENTRIES IN DISCRETE SERIES REPRESENTATIONS OF $s u(1,1)$}

For a (positive, or negative) discrete series representation $\pi$ of $s u(1,1)$, the operator

$$
T_{c}[\pi, c]:=\pi(-C)+c \cdot \pi(H)+\pi(B)
$$

is a tridiagonal operator for any $c \in \mathbb{R}$. Furthermore, the operators appearing in the $L D U$-decomposition of $T[\pi, c]$ are

$$
L[\pi]=\pi(-C), \quad D[\pi, c]=c \cdot \pi(H), \quad U[\pi]=\pi(B) .
$$


It is easy to see that $l_{n} u_{n} \neq 0$ holds in all cases, thus we may apply corollary 2.6 to compute $\left\langle e_{0},(\pi(-C)+c \cdot \pi(H)+\pi(B))^{m} e_{0}\right\rangle$ for every discrete series representation $\pi$, all $c \in \mathbb{R}$ and all $m \in \mathbb{N}$. In particular, using (1.15) and (1.16) we obtain following corollary.

Corollary 3.1. For any $k>0, m \in \mathbb{N}$ and $c \in \mathbb{R}$, the matrix entry $\left\langle e_{0},\left(\pi_{k}^{+}(-C)+\right.\right.$ $\left.\left.c \cdot \pi_{k}^{+}(H)+\pi_{k}^{+}(B)\right)^{m} e_{0}\right\rangle$ is the mth moment of the functional associated to the OPS $\left\{p[k, c]_{n}(x)\right\}_{n \geq 0}$ given by (1.1) and (1.2) where,

$$
b_{n}=2 c(k+n) \quad \text { and } \quad \lambda_{n}=n(n+2 k-1) .
$$

Similarly, the matrix entry $\left\langle e_{0},\left(\pi_{k}^{-}(-C)+c \cdot \pi_{k}^{-}(H)+\pi_{k}^{-}(B)\right)^{m} e_{0}\right\rangle$ is the mth moment of the functional associated to the $O P S\left\{p[k,-c]_{n}(x)\right\}_{n \geq 0}$.

The appropriate parts of the next proposition (restricted to the cases $|c|=1,|c|>1$ and $|c|<1$, respectively) are either implied or explicitly stated in some equivalent form in the works of Van Der Jeugt (1997), Koelink \& Van Der Jeugt (1998) and Groenevelt \& Koelink (2002). A detailed and deep simultaneous discussion of all three cases may be found in ch. 3 of Groenevelt's thesis (2004). Here we make a summary statement with respect to our bases and outline the proof that ensues from applying Meixner's classical method.

Proposition 3.2 (Groenevelt-Koelink-Van Der Jeugt). The OPS $\left\{p[k, c]_{n}(x)\right\}_{n \geq 0} d e-$ fined in corollary 3.1 is given by

$$
p[k, c]_{n}(x)= \begin{cases}\left(\sqrt{c^{2}-1}-c\right)^{n} m_{n}\left(\frac{x}{2 \sqrt{c^{2}-1}}-k ; 2 k, \frac{c-\sqrt{c^{2}-1}}{c+\sqrt{c^{2}-1}}\right) & \text { if }|c|>1, \\ L_{n}^{(2 k-1)}(x) & \text { if } c=1, \\ (-1)^{n} L_{n}^{(2 k-1)}(-x) & \text { if } c=-1, \\ \left(\sqrt{1-c^{2}}\right)^{n} \cdot M_{n}\left(x / \sqrt{1-c^{2}} ; c / \sqrt{1-c^{2}}, 2 k\right) & \text { if }|c|<1 .\end{cases}
$$

Here the polynomials $\left\{L_{n}^{(2 k-1)}(x)\right\}_{n \geq 0}$ are Laguerre polynomials, the polynomials $\left\{m_{n}(x ; 2 k,(c-\right.$ $\left.\left.\left.\sqrt{c^{2}-1}\right) /\left(c+\sqrt{c^{2}-1}\right)\right)\right\}_{n \geq 0}$ and $\left\{M_{n}\left(x ; c / \sqrt{1-c^{2}}, 2 k\right)\right\}_{n \geq 0}$, respectively, are Meixner polynomials of the first and second kind, respectively.

Proof. We apply Meixner's method to classify all OPS $\left\{P_{n}(x)\right\}_{n \geq 0}$ given by (1.1) and (1.2) where

$$
b_{n}=d n+f \quad \text { and } \quad \lambda_{n}=n(g n+h),
$$

see section 3 of ch. 6 in Chihara (1978). By (3.2) we have

$$
d=2 c, \quad f=2 c k, \quad g=1, \quad h=2 k-1 .
$$

The case $d^{2}-4 g=4\left(c^{2}-1\right)>0$ is thus equivalent to $|c|>1$. Following section 3 of ch. 6 in Chihara (1978) we set $\rho:=\sqrt{d^{2}-4 g}=2 \sqrt{c^{2}-1}$. Note next that replacing $p[k, c]_{n}(x)$ with

$$
q[k, c]_{n}(x):=p[k, c]_{n}\left(x-\gamma_{k, c}\right)
$$

yields an OPS $\left\{q_{n}[k, c](x)\right\}_{n \geq 0}$ satisfying (3.3) with

$$
d=2 c, \quad f=2 c k+\gamma_{k, c}, \quad g=1, \quad h=2 k-1 .
$$


We want to choose $\gamma_{k, c}$ in such a way that

$$
f=\frac{2(g+h)}{d+\rho}, \quad \text { i.e., } \quad 2 c k+\gamma_{k, c}=\frac{4 k}{2 c+2 \sqrt{c^{2}-1}}
$$

is satisfied. This yields

$$
\gamma_{k, c}=\frac{2 k}{c+\sqrt{c^{2}-1}}-2 k c=2 k\left(c-\sqrt{c^{2}-1}\right)-2 k c=-2 k \sqrt{c^{2}-1}
$$

and we may apply the results stated in section 3 of ch. 6 in Chihara (1978) to $\left\{q_{n}[k, c](x)\right\}_{n \geq 0}$. The parameter " $c$ " appearing in the definition of the corresponding Meixner polynomials of the first kind becomes

$$
\frac{d-\rho}{d+\rho}=\frac{2 c-2 \sqrt{c^{2}-1}}{2 c+2 \sqrt{c^{2}-1}}=\frac{c-\sqrt{c^{2}-1}}{c+\sqrt{c^{2}-1}} .
$$

and the parameter $\beta$ becomes $1+(2 k-1) / 1=2 k$. We obtain

$$
\left(\frac{1}{\sqrt{c^{2}-1}-c}\right)^{n} q[k, c]_{n}\left(2 \sqrt{c^{2}-1} x\right)=m_{n}\left(x ; 2 k,\left(c-\sqrt{c^{2}-1}\right) /\left(c+\sqrt{c^{2}-1}\right)\right)
$$

or, equivalently,

$$
q[k, c]_{n}(x)=\left(\sqrt{c^{2}-1}-c\right)^{n} m_{n}\left(\frac{x}{2 \sqrt{c^{2}-1}} ; 2 k, \frac{c-\sqrt{c^{2}-1}}{c+\sqrt{c^{2}-1}}\right) .
$$

The formula for $p[k, c]_{n}(x)$ now follows from $p[k, c]_{n}(x)=q[k, c]_{n}\left(x+\gamma_{k, c}\right)$.

The case $d^{2}-4 g=4\left(c^{2}-1\right)<0$ is equivalent to $|c|<1$. The parameter $\delta$ of the corresponding Meixner polynomials of the second kind becomes $2 c / \sqrt{4-4 c^{2}}=$ $c / \sqrt{1-c^{2}}$ and the parameter $\eta$ becomes $1+(2 k-1) / 1=2 k$. We obtain

$$
\left(\frac{1}{\sqrt{1-c^{2}}}\right)^{n} p[k, c]_{n}\left(x \sqrt{1-c^{2}}\right)=M_{n}\left(x ; c / \sqrt{1-c^{2}}, 2 k\right) .
$$

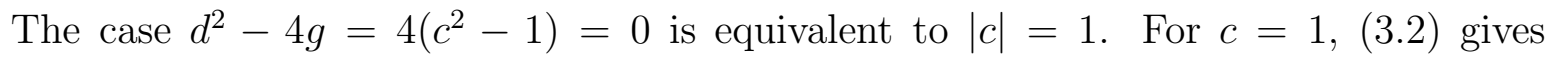
$b_{n}=2 n+2 k$ and $\lambda_{n}=n(n+2 k-1)$, which define the monic Laguerre polynomials $p[k, 1]_{n}(x)=L_{n}^{(2 k-1)}(x)$. Similarly, we also have $p[k,-1]_{n}(x)=(-1)^{n} L_{n}^{(2 k-1)}(-x)$.

Remark 3.3. Inspired by theorem 1.5 and corollary 1.4, for $|c|<1$ we may introduce a $\phi$ such that $0<\phi<\pi$ and $c=\cos (\phi)$ hold. Then we obtain

$$
p[k, \cos (\phi)]_{n}(x)=\sin (\phi)^{n} M_{n}(x / \sin (\phi) ; \cot (\phi), 2 k) .
$$

As indicated in the preliminaries, a combinatorial interpretation of the "Laguerre case" $|c|=1$ may be obtained using Viennot's (1983) "Laguerre stories". The "best" combinatorial interpretations (linked to permutation enumeration with no special weights) is associated to the case $k \in\{0,1\}$. At the light of corollary 1.6, this means that a combinatorial study of the matrix entries of the operator $R+L+S$ appearing in the work of Sukumar \& Hodges (2007) is easiest when $(\alpha-1) / 2$ or $(\alpha+1) / 2$ belongs to $\{0,1\}$. As a consequence of (1.6) we obtain

$$
\left\langle e_{0},(L+S+R)^{n} e_{0}\right\rangle=\left(\frac{\alpha+1}{2}\right)_{n} \text { for } \alpha \in(-1,1] .
$$


The case $\alpha=-1$ may be handled using remark 1.7. Similarly, for $e_{1}$ we have

$$
\left\langle e_{1},(L+S+R)^{n} e_{1}\right\rangle=\left(\frac{\alpha+3}{2}\right)_{n} \text { for all } \alpha \in[-1,1] .
$$

Another combinatorially interesting case is $c=0$ where proposition 3.2 gives $p[k, 0]_{n}(x)=$ $M_{n}(x ; 0,2 k)$. As a consequence, the operator $L+R$ appearing in the work of Sukumar \& Hodges (2007) satisfies

$$
\left\langle e_{0},(L+R)^{2 n} e_{0}\right\rangle=\mu_{2 n}(0,(\alpha+1) / 2) \text { for } \alpha \in(-1,1] .
$$

The case $\alpha=-1$ may again be handled using remark 1.7. Similarly, for $e_{1}$ we have

$$
\left\langle e_{1},(L+R)^{2 n} e_{1}\right\rangle=\mu_{2 n}(0,(\alpha+3) / 2) \text { for all } \alpha \in[-1,1] .
$$

Here $\mu_{2 n}(0, \eta)$ stands for the $(2 n)$ th moment associated to the Meixner polynomials of the second kind with parameters $(0, \eta)$. For $\eta=1$, (1.10) takes a very simple form, as this is noted in Example 21 of ch. II by Viennot (1983). Thus, by applying corollary 3.1 to $c=0$ and $k=1 / 2$, we obtain a combinatorial explanation for the first half of (1.22).

Remark 3.4. Viennot's (1983) work offers a nice model for the moments $\mu_{2 n}(0,1)$ by further restricting the appropriate "Laguerre stories". This corresponds to enumerating certain zig-zag permutations. For other values of $\eta$ we could not avoid avoid considering "weighted stories" in Viennot's (1983) model. The case $\alpha=0$ in the work Sukumar \& Hodges (2007) does not correspond to $\eta=1$, yet Sukumar \& Hodges (2007) find a simple, non-weighted model by considering the enumeration of transposition zigzag classes. It is an interesting question for future research whether this enumeration question may be related to Viennot's (1983) theory by constructing a highly nontrivial bijection, similarly to Viennot's (1983) bijection between all permutations and his "Laguerre stories".

For other values of $\eta$ we may use lemma 1.3 which gives

$$
\left\langle e_{0}, \pi_{k}^{+}(B-C)^{m} e_{0}\right\rangle=E_{2 m}^{(2 k)} \quad \text { where } \quad \sec ^{2 k}(t)=\sum_{n \geq 0} E_{n}^{(2 k)} \frac{t^{n}}{n !}
$$

In particular, substituting $k=1$ into (3.9) yields the second half of (1.22). Indeed, as noted in eq. (3.2) of Sukumar \& Hodges (2007), we have $E_{n}^{(2)}=T_{2 n+1}$. Using (3.9) for an arbitrary parameter $k$ allows us to rewrite (3.7) and (3.8) as

$$
\begin{array}{cc}
\left\langle e_{0},(L+R)^{2 n} e_{0}\right\rangle=E_{2 n}^{((\alpha+1) / 2)} & \text { for } \alpha \in(-1,1] \text { and } \\
\left\langle e_{1},(L+R)^{2 n} e_{1}\right\rangle=E_{2 n}^{((\alpha+3) / 2)} & \text { for all } \alpha \in[-1,1] .
\end{array}
$$

Finally, in the case $|c|>1$, leading to Meixner polynomials of the first kind, we may use (1.8) to find $\left\langle e_{0},\left(\pi_{k}^{+}(-C)+c \cdot \pi_{k}^{+}(H)+\pi_{k}^{+}(B)\right)^{m} e_{0}\right\rangle$. We omit the details for this case and mention only one, combinatorially interesting, subcase. For $k=1 / 2$ and $c=3 / \sqrt{8}$ the parameter $\beta$ of the associated Meixner polynomial becomes 1 , whereas the parameter " $c$ " becomes $1 / 2$. As it is explained in ch. II, $\S 8$ of Viennot's work (1983), the unitary Meixner polynomials of the first kind $\left\{\hat{m}_{n}(x ; 1,1 / 2)\right\}_{n \geq 0}$ are the 
Kreweras polynomials whose moments $\mu_{n}$ are the numbers of bicolored permutations of $\{1,2, \ldots, n\}$ where the two colors are used to color the descents of each permutation.

What we have seen thus far is that the application of corollary 2.6 to positive discrete series representations of $s u(1,1)$ is linked to the use of Laguerre polynomials and of Meixner polynomials of both kinds. The same orthogonal polynomial sequences need to be used to apply theorem 1.2 to the same representations. The only difference is that, in each case, instead of the moments we would need to use the inverse polynomials of the same OPS. We may find these inverse polynomials using (1.13) and (1.14). For brevity's sake, in the rest of the section we only outline how to find the inverse polynomials of Laguerre and Meixner polynomials, and leave the adaptation of these data to theorem 1.2 to the reader.

The Laguerre polynomials $\left\{L_{n}^{(\alpha)}(x)\right\}_{n \geq 0}$ satisfy (29) in ch. II of Viennot's (1983) work:

$$
\sum_{n \geq 0} L_{n}^{(\alpha)}(x) \frac{t^{n}}{n !}=(1+t)^{-\alpha-1} \exp \left(x \frac{t}{1+t}\right),
$$

thus we must set $f(t)=(1+t)^{-\alpha-1}$ and $g(t)=t /(1+t)$ in (1.13). As indicated in table 4 of ch. III by Viennot (1983), this implies $g^{\langle-1\rangle}(t)=t /(1-t)$. Thus the inverse polynomials of $\left\{L_{n}^{(\alpha)}(x)\right\}_{n \geq 0}$ are given by

$$
\sum_{n \geq 0} Q_{n}(x) \frac{t^{n}}{n !}=\left(\frac{1}{1-t}\right)^{\alpha+1} \exp \left(x \frac{t}{1-t}\right) .
$$

Hence we have

$$
\begin{aligned}
\mu_{n, d} & =\left[x^{d}\right] Q_{n}(x)=\frac{n !}{d !} \sum_{j=0}^{n-d}(-1)^{j}\left(\begin{array}{c}
-\alpha-1 \\
j
\end{array}\right)\left(\begin{array}{c}
-d \\
n-d-j
\end{array}\right)(-1)^{n-d-j} \\
& =\frac{(-1)^{n-d} n !}{d !}\left(\begin{array}{c}
-d-\alpha-1 \\
n-d
\end{array}\right)
\end{aligned}
$$

implying

$$
\mu_{n, d}=\left(\begin{array}{l}
n \\
d
\end{array}\right)(\alpha+1+d)_{n-d}
$$

The generating function for the unitary Meixner polynomials $\left\{\hat{m}_{n}(x ; \beta, c)\right\}_{n \geq 0}$ may be found in table 4 of ch. III by Viennot (1983):

$$
\sum_{n \geq 0} \hat{m}_{n}(x ; \beta, c) \frac{t^{n}}{n !}=\left(1+\frac{t c}{1-c}\right)^{-\beta}\left(\frac{1-c+t}{1-c+c t}\right)^{x}
$$

thus we must set $f(t)=(1+t c /(1-c))^{-\beta}$ and $g(t)=\ln (1-t+c)-\ln (1-c+c t)$ in (1.13). As indicated in table 4 of ch. III by Viennot (1983), this implies $g^{\langle-1\rangle}(t)=$ $(1-c)(\exp (t)-1) /(1-c \exp (t))$, and the inverse polynomials of $\left\{\hat{m}_{n}(x ; \beta, c)\right\}_{n \geq 0}$ are 
given by

$$
\sum_{n \geq 0} Q_{n}(x) \frac{t^{n}}{n !}=\left(\frac{1-c}{1-c \exp (t)}\right)^{\beta} \exp \left(\frac{x(1-c)(\exp (t)-1)}{1-c \exp (t)}\right) .
$$

The Meixner polynomials of the second kind $\left\{M_{n}(x ;, \delta, \eta)\right\}_{n \geq 0}$ satisfy (69) in ch. II of Viennot's (1983) work:

$$
\sum_{n \geq 0} M_{n}(x ; \delta, \eta) \frac{t^{n}}{n !}=\left((1+\delta t)^{2}+t^{2}\right)^{-\eta / 2} \exp \left(x \arctan \left(\frac{t}{1+\delta t}\right)\right),
$$

thus we must set $f(t)=\left((1+\delta t)^{2}+t^{2}\right)^{-\eta / 2}$ and $g(t)=\arctan (t /(1+\delta t))$ in (1.13). As indicated in table 4 of ch. III by Viennot (1983), this implies $g^{\langle-1\rangle}(t)=\tan (t) /(1-$ $\delta \tan t)$, and the inverse polynomials of $\left\{M_{n}(x ;, \delta, \eta)\right\}_{n \geq 0}$ are given by

$$
\sum_{n \geq 0} Q_{n}(x) \frac{t^{n}}{n !}=\left(\frac{\sec (t)^{2}}{(1-\delta \tan (t))^{2}}\right)^{\eta / 2} \exp \left(x \frac{\tan (t)}{1-\delta \tan (t)}\right) .
$$

In particular, we have

$$
\mu_{n, d}=\frac{n !}{d !}\left[t^{n}\right] \sec (t)^{\eta} \tan (t)^{d} \quad \text { for } \delta=0
$$

\section{EigenVectors of ClOSABle tridiagonal operators}

In this section we return to general tridiagonal operators on the complex Hilbert space $\ell^{2}\left(\mathbb{Z}_{\geq 0}\right)$, but it will be convenient to assume that they are closable. This assumption was not necessary in section 2 , where we were only interested in calculating the matrix entries in the first column for the powers of tridiagonal operators. Formally, we are still able to state theorem 4.1 below without assuming closability. However, the theorem implies that the eigenvectors of tridiagonal operators of the stated form must arise as infinite linear combinations of the basis vectors. In all applications, it seems easier to show that such vectors belong to the domain of the operator when the operator is closable.

As noted at the end of section 2.1 by Groenevelt \& Koelink (2002), for any (positive or negative) discrete series representation $\pi$ of $s u(1,1)$, the operators $\pi(B), \pi(C)$ and $\pi(H)$ are closable. As a consequence, the operators operators $T_{c}$ defined in (3.1) are closable. They are even self-adjoint, i.e., Jacobi operators. We refer the reader to Koelink (2004) for the spectral theory of Jacobi operators. This theory was used by Van Der Jeugt (1997), Koelink \& Van Der Jeugt (1998) and Groenevelt \& Koelink (2002) to obtain a complete description of the spectra of the operators $T_{c}$. The main theorem of this section generalizes only the necessity part of their results. Even this generalization suffices to highlight the fact: the (potential) eigenvectors of any (closable) tridiagonal operator $T$ may be expressed using the same OPS, whose moments and and inverse polynomial coefficients may be used to express the first columns in the matrices of powers of $T$. 
Theorem 4.1. Let $T$ be a tridiagonal operator with $L D U$-decomposition $T=L+D+U$ such that $l_{n} u_{n} \neq 0$ holds for all $n \geq 0$ and let $z \in \mathbb{C}$ any complex number. If $z$ is an eigenvalue of $T$ then the associated eigenspace is one dimensional, generated by

$$
v_{z}:=\sum_{n \geq 0} \frac{p_{n}(z)}{\prod_{i=0}^{n-1} u_{i}} e_{n} .
$$

Here $\left\{p_{n}(x)\right\}_{n \geq 0}$ is the sequence of monic polynomials defined by (1.1) and (1.2), satisfying $b_{n}=d_{n}$ and $\lambda_{n}=u_{n-1} l_{n-1}$.

Proof. A vector of the form $\sum_{n \geq 0} h_{n} \cdot e_{n}$ is eigenvector of $T$ associated to the eigenvalue $z$ only if

$$
\begin{gathered}
d_{0} h_{0}+u_{0} h_{1}=z h_{0} \quad \text { and } \\
l_{n-1} h_{n-1}+d_{n} h_{n}+u_{n} h_{n+1}=z h_{n} \quad \text { holds for } n \geq 1 .
\end{gathered}
$$

Introducing $p_{0}:=h_{0}$ and

$$
p_{n}:=h_{n} \cdot u_{0} u_{1} \cdots u_{n-1} \quad \text { for } n \geq 1
$$

we may rewrite (4.1) as

$$
p_{1}=\left(z-d_{0}\right) p_{0}
$$

while multiplying both sides of (4.2) by $u_{0} u_{1} \cdots u_{n-1}$ yields the equivalent equation

$$
p_{n+1}=\left(z-d_{n}\right) p_{n}-u_{n-1} l_{n-1} p_{n-1} \quad \text { for } n \geq 1 \text {. }
$$

If $p_{0}=0$ then (4.3) and (4.4) implies $p_{n}=0$ for all $n$. We obtain a nonzero eigenvector only if $p_{0} \neq 0$ and then, without loss of generality we may assume $p_{0}=1$. Furthermore, (4.4) implies that $p_{n}$ must be obtained by substituting $z$ into a monic polynomial sequence $\left\{p_{n}(x)\right\}_{n \geq 0}$ satisfying Favard's recursion formula with $b_{n}=d_{n}$ and $\lambda_{n}=$ $u_{n-1} l_{n-1}$ for $n \geq 1$. Therefore any eigenvector associated to $z$ is of the form stated in the theorem.

Remark 4.2. In the special case when the tridiagonal operator $T$ is a Jacobi operator, $T$ is self-adjoint and thus has only real eigenvalues.

The connecting coefficients $\left\{\lambda_{n}\right\}_{n \geq 1}$ resp. $\left\{b_{n}\right\}_{n \geq 0}$ for the polynomials $\left\{p_{n}(x)\right\}_{n \geq 0}$ used in theorem 4.1 above are the same as the ones used in corollary 2.6 and theorem 2.8. Thus, the same OPS may be used to calculate the first column of matrix entries of the powers of $T$ as in the calculation of its eigenvectors. In particular, for any operator $T_{c}$ defined in (3.1) we must use the appropriate OPS $\left\{p[k, c]_{n}(x)\right\}_{n \geq 0}$ from proposition 3.2. This leads to the use of Laguerre polynomials, and Meixner polynomials of both kinds, as in the above cited results of Van Der Jeugt (1997), Koelink \& Van Der Jeugt (1998) and Groenevelt \& Koelink (2002).

We conclude this section with an observation regarding the case $c=0$ and $k=1 / 2$, leading to $p[1 / 2,0](x)=M_{n}(x ; 0,1)$, by proposition 3.2. A combinatorial model for the coefficients of the powers of $x$ in $M_{n}(x ; 0,1)$ is mentioned in Example 21 of ch. II by Viennot (1983). Up to sign, the coefficient of $x^{j}$ in $M_{n}(x ; 0,1)$ is the number of permutations of $\{1,2, \ldots, n\}$ having $j$ odd cycles. Thus, for this specific choice 
of parameters, not only the selected matrix entries of the powers of $T_{0}$, but also the description of the eigenvectors has a combinatorial interpretation. Recall that the same selection of parameters leads to the first part of (1.22), which is equivalent to the first part of (1.21) that also caught the attention of Hodges \& Sukumar (2007). Therefore, if someone wanted to build a "purely combinatorial model" of a quantum oscillator in the future, starting with $\alpha=1$ in the model proposed Hodges \& Sukumar (2007) or with $k=1 / 2$ in the model proposed by Klimyk (2006), and focusing on understanding the action of the moment operator seems a very reasonable first step.

\section{BEYOND SELF-ADJOINT OPERATORS AND BEYOND $s u(1,1)$}

Up to this point, our applications of theorems [2.5, 2.8 and 4.1 involved self-adjoint operators from discrete series representations of $s u(1,1)$. In this final section we outline two examples indicating that that these theorems may also be useful in other applications where the operator is not self-adjoint or where it belongs to the representation of an algebra that is is not $s u(1,1)$.

Our first example is $\pi_{k}^{+}(B+C)$ where $\pi_{k}^{+}$is a positive discrete series representation of $s u(1,1)$ for some $k>0$. In analogy to corollary 3.1, the matrix entry $\left\langle e_{0}, \pi_{k}^{+}(B+C)^{m} e_{0}\right\rangle$ is the $m$ th moment of the functional associated to the OPS $\left\{f_{n}^{(2 k)}(x)\right\}_{n \geq 0}$ given by (1.1) and (1.2) where

$$
b_{n}=0 \quad \text { and } \quad \lambda_{n}=-n(n+2 k-1) .
$$

It is easy to verify that we have

$$
f_{n}^{(2 k)}(x)=(-\mathbf{i})^{n} M_{n}(\mathbf{i} x ; 0,2 k)
$$

where the polynomials $M_{n}(x ; 0,2 k)$ are Meixner polynomials of the second kind. Thus,

for $k \in \frac{1}{2} \mathbb{Z}_{\geq 0}$, the polynomials $f_{n}^{(2 k)}(x)$ are identical to the polynomials studied by Carlitz (1959). The applications of theorems 2.5 and 2.8 are no different from the selfadjoint setting. By theorem 4.1, a complex number $z \in \mathbb{C}$ is an eigenvalue $\pi_{k}^{+}(B+C)$ only if the expression

$$
v_{z}^{(k)}:=\sum_{n \geq 0} \frac{f_{n}^{(2 k)}(z)}{\sqrt{(n-1) !(2 k)_{n}}} e_{n}
$$

represents a vector belonging to $\ell^{2}\left(\mathbb{Z}_{\geq 0}\right)$. The the discussion of the convergence issues is analogous to the case of $\pi_{k}^{+}(B-C)$ that was worked out by Koelink \& Van Der Jeugt (1998): here we obtain that all eigenvalues belong to $\mathbf{i} \mathbb{R}$. This is not surprising, since $\mathbf{i} \pi_{k}^{+}(B+C)$ is self-adjoint. However, the way were able to handle the example indicates that the validity of our main results is not restricted to self-adjoint operators.

Our second example is the sum $A+A^{\dagger}$ where $A$ and $A^{\dagger}$ are the operators defined in eq. (2.1) of Sukumar \& Hodges (2007):

$$
\begin{array}{lll}
A e_{2 n}=\sqrt{2 n} \cdot e_{2 n-1}, & A e_{2 n+1}=\sqrt{2 n+1+\alpha} \cdot e_{2 n}, \\
A^{\dagger} e_{2 n}=\sqrt{2 n+1+\alpha} \cdot e_{2 n+1}, & A^{\dagger} e_{2 n-1}=\sqrt{2 n} \cdot e_{2 n} .
\end{array}
$$


The operator $A+A^{\dagger}$ is self-adjoint, but it belongs to a representation of a Lie superalgebra properly containing $s u(1,1)$. Theorems 2.5, 2.8 and 4.1 are obviously applicable, and the OPS $\left\{p_{n}(x)\right\}_{n \geq 0}$ appearing in all of them is given by (1.1) and (1.2) where,

$$
b_{n}=0, \quad \lambda_{2 n+1}=2 n+1+\alpha, \quad \text { and } \quad \lambda_{2 n}=2 n .
$$

We obtain the generalized Hermite polynomials, see eq. (2.46) of ch. V in Chihara (1978). Choosing $\alpha=0$ yields the usual Hermite polynomials. Sukumar \& Hodges (2007) note that this choice corresponds to the standard harmonic oscillator. For nonzero $\alpha$, the parity-dependent nature of the quantum algebra defined by Sukumar \& Hodges (2007) is reflected in the parity-dependent nature of the recursion defining the generalized Hermite polynomials.

\section{ACKNOWLEDGEMENTS}

I wish to thank Mireille Bousquet-Mélou for acquainting me with Viennot's (1983) work and two anonymous referees whose suggestions greatly improved the content and presentation of this paper. Many thanks to Erik Koelink and Alan Lambert who promptly answered many of my questions. This work was supported by the NSA grant \# H98230-07-1-0073 and essentially completed while the author was on reassignment of duties sponsored by the University of North Carolina at Charlotte.

\section{REFERENCES}

[1] Carlitz, L. 1959 Bernoulli and Euler numbers and orthogonal polynomials. Duke Math. J. 26, $1-16$.

[2] Chihara, T. S. 1978 An introduction to orthogonal polynomials. New York-London-Paris: Gordon and Breach Science Publishers.

[3] Groenevelt, W. 2004 Tensor product representations and special functions, doctoral thesis, Delft University Press.

[4] Groenevelt, W. \& Koelink, E. 2002 Meixner functions and polynomials related to Lie algebra representations, J. Phys. A 35, 65-85.

[5] Hodges, A. \& Sukumar, C. V. 2007 Bernoulli, Euler, permutations and quantum algebras. Proc. R. Soc. A 463, 2401-2414.

[6] Klimyk, A. U. 2006 The su(1,1)-models of quantum oscillator. Ukr. J. Phys. 51, 1019-1027.

[7] Koekoek, R. \& Swarttouw, R. F. 1998 The Askey-scheme of hypergeometric orthogonal polynomials and its $q$-analogue, http://fa.its.tudelft.nl/ koekoek/askey/

[8] Koelink, E. 2004 Spectral theory and special functions, Laredo lectures on orthogonal polynomials and special functions, 45-84, Adv. Theory Spec. Funct. Orthogonal Polynomials, Hauppage, NY: Nova Sci. Publ.

[9] Koelink, E. \& Van Der Jeugt, J. 1998, Convolutions for orthogonal polynomials from Lie and quantum algebra representations, SIAM J. Math. Anal. 29, 794-822.

[10] Stanton, D. 2003, Enumeration and special functions, in "Orthogonal polynomials and special functions (Leuven 2002)", 137-166, Lecture Notes in Math. 1817, Berlin: Springer.

[11] Sukumar, C. V. \& Hodges, A. 2007 Quantum algebras and parity-dependent spectra, Proc. R. Soc. A 463, 2415-2427.

[12] Van Der Jeugt, J. 1997 Coupling coefficients for Lie algebra representations and addition formulas for special functions, J. Math. Phys. 38, 2728-2740. 
[13] Viennot, X. 1983 Une théorie combinatoire des polynômes orthogonaux généraux. Montreal: Lecture Notes LACIM, UQAM, 219p. Publication du LACIM, Université du Québec à Montréal, 1984, réed. 1991. Available online at the author's personal website http://web.mac . com/xgviennot

[14] Vilenkin, N. Ja. \& Klimyk, A. U. 1991 Representation of Lie groups and special functions, vol. 1. Dordrecht: Kluwer.

E-mail address: ghetyei@uncc.edu

Department of Mathematics and Statistics, University of North Carolina at CharLotte, Charlotte, NC 28223, USA 